\title{
Studies of a New-style Resonator on Electro- mechanical Coupling Bandgap Control of a Locally Resonant Piezoelectric/elastic Phononic Crystal Double-layer Nonlocal Nanobeam
}

\author{
Denghui Qian ( $\nabla$ dhqian@just.edu.cn ) \\ Jian Zhang \\ Jiangsu University of Science and Technology \\ Jianchun Wang \\ China Ship Scientific Research Center
}

Jiangsu University of Science and Technology https://orcid.org/0000-0001-5516-6831

\section{Original Article}

Keywords: locally resonant piezoelectric/elastic phononic crystal double layer nanobeam, nonlocal piezoelectricity theory, plane wave expansion method, symmetric and antisymmetric modes, nano electro mechanical system

Posted Date: May 20th, 2021

DOI: https://doi.org/10.21203/rs.3.rs-520315/v1

License: (c) (i) This work is licensed under a Creative Commons Attribution 4.0 International License. Read Full License

Version of Record: A version of this preprint was published at Applied Mathematical Modelling on February 1st, 2022. See the published version at https://doi.org/10.1016/j.apm.2021.10.020. 
Studies of a new-style resonator on electro-mechanical coupling bandgap control of a locally resonant piezoelectric/elastic phononic crystal double-layer nonlocal nanobeam Denghui Qian, Zhang Jian

School of Naval Architecture \& Ocean Engineering, Jiangsu University of Science and Technology, Zhenjiang 212100, Jiangsu, China

e-mail:dhqian@just.edu.cn

\section{Jianchun Wang}

China Ship Scientific Research Center, Wuxi 214082, Jiangsu, China

\section{ABSTRACT}

The paper proposed a model of a locally resonant (LR) piezoelectric/elastic phononic crystal (PC) nanobeam with periodically attached "spring-mass" resonator and additional spring between upper and lower nanobeams, as well as horizontal spring between mass and foundation. Euler beam theory and nonlocal piezoelectricity theory are coupled and introduced to plane wave expansion (PWE) method to calculate the band structures of such a model with different parameters. Numerical results and further analysis demonstrate that all the bands of double-layer nanobeam can be divided into symmetric and antisymmetric ones. Adding additional and horizontal springs play a role in control the symmetric and antisymmetric bands respectively, which make wider band gaps be opened than corresponding single-layer nanobeam. Moreover, the change of parameters of electro-mechanical coupling fields and resonator can be applied to effectively control the starting frequencies and widths of band gaps, which can provide a theoretical basis for active control of vibration. Effects of geometric and non-dimensional nonlocal parameters on band gaps are also discussed. All the studies are expected to be applied to actively control vibration propagation in the field of nano electro-mechanical system (NEMS).

Key words: locally resonant piezoelectric/elastic phononic crystal double-layer nanobeam; nonlocal piezoelectricity theory; plane wave expansion method; symmetric and antisymmetric modes; nano electro-mechanical system

\section{INTRODUCTION}

Recently, different kinds of NEMS have been widely applied to national defense, civil use, biomedicine and so on, which have excellent characteristics such as small size, superhigh frequency, lower power, high sensitivity and so on. The existing piezoelectric nano devices include biological motor, resonant sensor, filter and so on ${ }^{[1]}$. As for artificial periodic composite structures, PCs in various forms have attracted wide attentions on account of the existing of band gaps, which have been applied to vibration and noise control, acoustics lens, acoustical devices and so on ${ }^{[2]}$. Generally, PCs can be divided to Bragg scattering and LR types. Particularly, the opening of band gaps of LR PCs is mainly based on the locally resonance of scatters, which have an advantage in opening band gaps in low frequency region ${ }^{[3]}$. With the rapid development of industry, double-layer structures with the multifunctional characteristics, such as light weight, large stiffnes, good impact resistance and so on, have been widely used in the field of naval architecture, aeronautics, astronautics, civil engineering and so on ${ }^{[4]}$. Moreover, the cavity of a double-layer structure in engineering is generally periodically added sandwich to enhance structural performance, which makes the double-layer structures have an excellent convergence point with $\mathrm{PCs}^{[5]}$. Hence, based on the common application background in acoustical devices of NEMS and PCs, excellent characteristics of piezoelectric nanostructures, PCs and double-layer structures will be gathered, and the new coupling physical characteristics will be displayed if the 
design ideas of them are coupled and introduced to double-layer structures.

As far as it goes, the piezoelectric PC nanostructures were directly studied slightly. By introducing nonlocal piezoelectricity theory to transfer matrix (TM) method, the plane ${ }^{[6]}$, symmetric $^{[7]}$ and anti-plane transverse wave $\operatorname{modes}^{[8]}$ of one-dimensional (1D) layered piezoelectric PC nanostructures were studied by Yan, Chen and Wang et al.. Based on PWE method, the band structures of two-dimensional (2D) piezoelectric PC nanostructures with different types of scatters and lattices were calculate and corresponding bandgap properties were researched by Miranda Jr and Dos Santos ${ }^{[9]}$. By applying PWE method, the effects of nonlocal effects $^{[10]}$, surface effects ${ }^{[11]}$, nonlinearity ${ }^{[12]}$ and locally resonance ${ }^{[13]}$ on electro-mechanical coupling wave propagation characteristics of piezoelectric PC nanobeams were investigated by Qian et al.. Consequently, deep researches are needed for piezoelectric PC nanostructures.

In recent years, the dynamic characteristics of piezoelectric nanostructures and wave propagation properties of PC structures have obtained wide studies. In order to overcome the size dependence existing in nanostructures, classical continuum elasticity theory has been revised to form several higher-order continuum elasticity theories ${ }^{[6,11,14,15]}$. As for the common theory, nonlocal elasticity theory has been further developed to nonlocal piezoelectricity theory to study the mechanical characteristics of piezoelectric nanostructures ${ }^{[16,17]}$. PC structures at macroscopical size, proposed in thirty years ago, have been widely introduced to some basic elastic structures in engineering such as beam, plate and so on ${ }^{[18,19]}$. In order to overcome the disadvantage that the frequency ranges of band gaps are fixed if the PC structures are manufactured, multi-physics coupling PC structures have been proposed, such as: piezoelectric, piezomagnetic, magneto-electro-elastic PCs and so on ${ }^{[20-22]}$. Particularly, band gaps can be effectively controlled to piezoelectric PCs by converting the electric and mechanical fields. Moreover, with the rapid development of nanotechnology in different fields, PC structures at nanoscale have been proposed and studied, which can make the order of magnitude of bandgap frequency range sharply increase to be superhigh (gigahertz $(\mathrm{GHz})$ even terahertz $(\mathrm{THz}))^{[23,24]}$.

On the basis of existing researches on different PC structures and piezoelectric nanostructures, PWE method is extended to calculate the band structure of a proposed LR piezoelectric/elastic PC Euler double-layer nonlocal nanobeam with horizontal and additional springs periodically attached in this paper. Moreover, in order to reveal the unique tunability of such a new-style resonator on band gaps, the formation mechanisms and influence rules on band gaps of first two band gaps are studied in detail.

\section{MODEL AND METHOD}

As shown in Fig. 1, the proposed LR piezoelectric/elastic PC double-layer nanobeam is composed of two parts: base double-layer nanobeam and resonator. The base double-layer nanobeam is formed by periodically repeating a piezoelectric material PZT-4 and an elastic material epoxy in the axial direction. The resonator is formed by four parts: mass $m_{\mathrm{R}}$, vertical spring $k_{z}$, horizontal spring $k_{y}$ and additional spring $k_{\mathrm{A}}$. In a resonator, the mass is connected by two vertical springs and two horizontal springs. Generally, the foundation hardly vibrated is occurred in a mechanical system. By periodically attaching the vertical springs and additional springs onto the midpoint of each PZT-4 part of double-layer nanobeam and the horizontal springs on to the foundation, such a model is formed. The Cartesian coordinate system is set up as shown in Fig. 1(b). Here, all the horizontal springs are located at $y$-direction when the model is balanced. The original length of each horizontal spring is $L_{y}$ and the length between mass and foundation is 
$L$. If $L<L_{y}$, each horizontal spring is pre-compressed in the equilibrium position. Each PZT-4 is applied by an external electrical voltage $V$ and each nanobeam is applied by an external axial force $P_{0}$. The lengths of each PZT-4 and epoxy are $a_{1}$ and $a_{2}$, respectively. The lattice constant of such a PC double-layer nanobeam is $a=a_{1}+a_{2}$. Besides, the width and thickness of each nanobeam with rectangular cross section are $b$ and $h$, respectively. As shown in the figure, the position of each resonator can be expressed as: $X=a_{1} / 2+\bar{m} a$, where $\bar{m}=0, \pm 1, \pm 2, \cdots$. Moreover, Table I gives all the material parameters of PZT-4 and epoxy used in the following calculations. In Table I, $\rho$ denotes the mass density, which can be further divided into $\rho_{1}$ and $\rho_{2}$ to expressed PZT-4 and epoxy, respectively. $E$ represents the elastic modulus of epoxy. $c_{11}, e_{31}$ and $\kappa_{33}$ are the elastic, piezoelectric and dielectric constants of PZT-4, respectively.

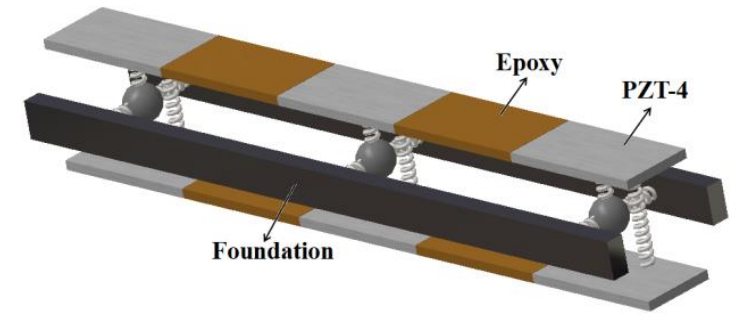

(a)

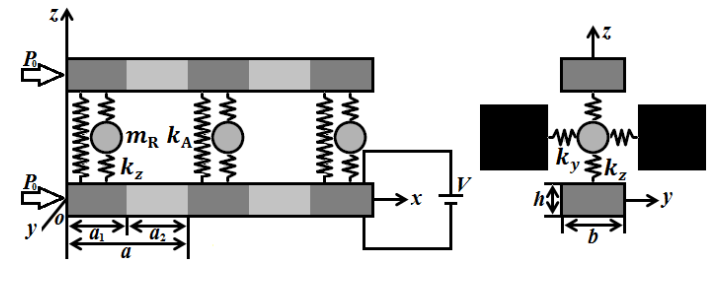

(b)

FIG. 1. (a) Physical model of a LR piezoelectric/elastic PC double-layer nanobeam, and (b) the corresponding computational model.

Table 1. Bulk material parameters of PZT-4 ${ }^{[10]}$ and epoxy.

\begin{tabular}{cccccc}
\hline Material & $\rho / \mathrm{kgm}^{-3}$ & $E / \mathrm{GPa}$ & $c_{11} / \mathrm{GPa}$ & $e_{31} / \mathrm{Cm}^{-2}$ & $\kappa_{33} / \mathrm{CV}^{-1} \mathrm{~m}^{-1}$ \\
\hline PZT-4 & 7500 & & 132 & -4.1 & $7.124 \times 10^{-9}$ \\
epoxy & 1180 & 4.35 & & & \\
\hline The time-harmonic & flexural & vibration & governing & equations of & proposed $\mathrm{LR}$
\end{tabular}

The time-harmonic flexural vibration governing equations of proposed LR piezoelectric/elastic PC Euler double-layer nonlocal nanobeam can be written as ${ }^{[10,11]}$ :

$$
\left\{\begin{array}{c}
\frac{\partial^{2}}{\partial x^{2}}\left(m(x) \frac{\partial^{2} W_{1}(x)}{\partial x^{2}}\right)-n(x) \frac{\partial^{2} W_{l}(x)}{\partial x^{2}}+\mu^{2} \frac{\partial^{2}}{\partial x^{2}}\left[n(x) \frac{\partial^{2} W_{l}(x)}{\partial x^{2}}\right] \\
-\omega^{2} o(x) W_{1}(x)+\omega^{2} \mu^{2} \frac{\partial^{2}}{\partial x^{2}}\left[o(x) W_{1}(x)\right]=\sum_{X} Q_{1}(X) \\
-\omega^{2} m_{\mathrm{R}} W_{\mathrm{R}}(X)=Q_{\mathrm{R}}(X) \\
\frac{\partial^{2}}{\partial x^{2}}\left(m(x) \frac{\partial^{2} W_{\mathrm{u}}(x)}{\partial x^{2}}\right)-n(x) \frac{\partial^{2} W_{\mathrm{u}}(x)}{\partial x^{2}}+\mu^{2} \frac{\partial^{2}}{\partial x^{2}}\left[n(x) \frac{\partial^{2} W_{\mathrm{u}}(x)}{\partial x^{2}}\right] \\
-\omega^{2} o(x) W_{\mathrm{u}}(x)+\omega^{2} \mu^{2} \frac{\partial^{2}}{\partial x^{2}}\left[o(x) W_{\mathrm{u}}(x)\right]=\sum_{X} Q_{\mathrm{u}}(X)
\end{array}\right.
$$

where

$$
\left\{\begin{array}{c}
Q_{\mathrm{l}}(X)=-k_{z}\left[W_{\mathrm{l}}(X)-W_{\mathrm{R}}(X)\right] \delta(x-X)-k_{\mathrm{A}}\left[W_{\mathrm{l}}(X)-W_{\mathrm{u}}(X)\right] \delta(x-X) \\
Q_{\mathrm{R}}(X)=k_{z}\left[W_{\mathrm{l}}(X)-2 W_{\mathrm{R}}(X)+W_{\mathrm{u}}(X)\right]+2 k_{y}\left[\frac{L_{y}}{\sqrt{W_{\mathrm{R}}^{2}(X)+L^{2}}}-1\right] W_{\mathrm{R}}(X) \\
Q_{\mathrm{u}}(X)=-k_{z}\left[W_{\mathrm{u}}(X)-W_{\mathrm{R}}(X)\right] \delta(x-X)-k_{\mathrm{A}}\left[W_{\mathrm{u}}(X)-W_{\mathrm{l}}(X)\right] \delta(x-X)
\end{array}\right.
$$

Here, $W_{1}(x)$ and $W_{\mathrm{u}}(x)$ are used to represent the flexural displacements of lower-layer and upper-layer nanobeams along $z$-direction, respectively. $W_{\mathrm{l}}(X)$ and $W_{\mathrm{u}}(X)$ are the flexural displacements of lower-layer and upper-layer nanobeams at $X$, respectively. $W_{\mathrm{R}}(X)$ is the displacement of mass along $z$-direction at $X . Q_{\mathrm{l}}(X)$ and $Q_{\mathrm{u}}(X)$ are the forces applied to the lower-layer and upper-layer nanobeams by vertical and additional springs at $X$, respectively. 
$Q_{\mathrm{R}}(X)$ is the force applied to the mass by vertical and horizontal springs at $X$. Moreover, $\delta(x-X)$ is the one-dimensional (1D) delta function and $\omega$ is the vibration frequency. $\mu$ is a nonlocal coefficient used to represent the nonlocal effects based on nonlocal piezoelectricity theory, and the derived parameter $\eta=\mu / a$ can be further applied to represent the ratio between nonlocal size and lattice constant ${ }^{[10]} . m(x), n(x)$ and $o(x)$ are used to uniformly express parameters $\left(m_{1}, m_{2}\right),\left(n_{1}, n_{2}\right)$ and $\left(o_{1}, o_{2}\right)$ in such a PC double-layer nanobeam, the details are as follows:

$$
\begin{gathered}
m_{1}=\frac{b h^{3}}{12} \bar{c} \\
n_{1}=P_{0}+b e_{31} V \\
o_{1}=\rho_{1} b h \\
m_{2}=\frac{b h^{3}}{12} E \\
n_{2}=P_{0} \\
o_{2}=\rho_{2} b h
\end{gathered}
$$

where $\bar{c}=c_{11}+e_{31}^{2} / \kappa_{33}$.

As shown in Eq. (2), the second item of $Q_{\mathrm{R}}(X)$ is nonlinear, which can be expanded by Fourier series and ignored second and higher order terms. Finally, Eq. (2) can be linearized as:

$\left\{\begin{array}{c}Q_{\mathrm{l}}(X)=-k_{z}\left[W_{\mathrm{l}}(X)-W_{\mathrm{R}}(X)\right] \delta(x-X)-k_{\mathrm{A}}\left[W_{\mathrm{l}}(X)-W_{\mathrm{u}}(X)\right] \delta(x-X) \\ Q_{\mathrm{R}}(X)=k_{z}\left[W_{\mathrm{l}}(X)-2 W_{\mathrm{R}}(X)+W_{\mathrm{u}}(X)\right]+2 k_{y}(l-1) W_{\mathrm{R}}(X) \\ Q_{\mathrm{u}}(X)=-k_{z}\left[W_{\mathrm{u}}(X)-W_{\mathrm{R}}(X)\right] \delta(x-X)-k_{\mathrm{A}}\left[W_{\mathrm{u}}(X)-W_{\mathrm{l}}(X)\right] \delta(x-X)\end{array}\right.$

where non-dimensional parameter $l=L_{y} / L$ is used to represent the degree of pre-compression.

Based on the periodicity of nanobeam in $x$-direction, $\xi(x)(\xi=m, n, o)$ can be expressed in spatial Fourier series as:

$$
\xi(x)=\sum_{G} \xi(G) e^{i G x}
$$

where $G$ is the $1 \mathrm{D}$ reciprocal-lattice vector. Here, $\xi(G)$ can be expressed as:

$$
\xi(G)=\left\{\begin{array}{lc}
\xi_{1} f+\xi_{2}(1-f), & G=0 \\
\left(\xi_{1}-\xi_{2}\right) \psi(G), & G \neq 0
\end{array}\right.
$$

where $f=a_{1} / a$ represents the filling ratio of PZT-4, and $\psi(G)=f \sin \left(G a_{1} / 2\right) /\left(G a_{1} / 2\right)$.

According to the Bloch theory and periodicity of model, $W_{\mathrm{l}}(x)$ and $W_{\mathrm{u}}(x)$ can be expressed as:

$$
\left\{\begin{array}{l}
W_{\mathrm{l}}(x)=\sum_{G^{\prime}} W_{\mathrm{l}}\left(G^{\prime}\right) e^{i\left(k+G^{\prime}\right) x} \\
W_{\mathrm{u}}(x)=\sum_{G^{\prime}} W_{\mathrm{u}}\left(G^{\prime}\right) e^{i\left(k+G^{\prime}\right) x}
\end{array}\right.
$$

where $k$ is the Bloch wave vector limited in the irreducible first Brillouin zone (1BZ), and $G^{\prime}$ is also the $1 \mathrm{D}$ reciprocal-lattice vector.

Moreover, the Bloch theory and periodicity of model imply that:

$$
\left\{\begin{array}{l}
W_{\mathrm{l}}(X)=W_{\mathrm{l}}(0) e^{i k X} \\
W_{\mathrm{R}}(X)=W_{\mathrm{R}}(0) e^{i k X} \\
W_{\mathrm{u}}(X)=W_{\mathrm{u}}(0) e^{i k X}
\end{array}\right.
$$

The delta function $\delta(x-X)$ suggests the following relations: 


$$
\left\{\begin{array}{c}
\sum_{X} e^{i k X} \delta(x-X)=e^{i k x} \sum_{X} \delta(x-X) \\
\sum_{X} \delta(x-X)=\frac{1}{a} \sum_{G^{\prime}} e^{i G^{\prime} x}
\end{array}\right.
$$

By substituting $x=0$ to Eq. (12), it obtains that:

$$
\left\{\begin{array}{l}
W_{\mathrm{l}}(0)=\sum_{G^{\prime \prime}} W_{\mathrm{l}}\left(G^{\prime \prime}\right) \\
W_{\mathrm{u}}(0)=\sum_{G^{\prime \prime}} W_{\mathrm{u}}\left(G^{\prime \prime}\right)
\end{array}\right.
$$

By substituting Eqs. (9), (10), (12)-(15) to (1), it gives:

$$
\left\{\begin{array}{c}
\sum_{G^{\prime}}\left\{\left(k+G^{\prime \prime}\right)^{2} m\left(G^{\prime \prime}-G^{\prime}\right)\left(k+G^{\prime}\right)^{2}+\left[1+\mu^{2}\left(k+G^{\prime \prime}\right)^{2}\right] n\left(G^{\prime \prime}-G^{\prime}\right)\left(k+G^{\prime}\right)^{2}\right\} W_{\mathrm{l}}\left(G^{\prime}\right) \\
-\omega^{2} \sum_{G^{\prime}}\left\{\left[1+\mu^{2}\left(k+G^{\prime \prime}\right)^{2}\right] o\left(G^{\prime \prime}-G^{\prime}\right)\right\} W_{\mathrm{l}}\left(G^{\prime}\right) \\
=-\frac{k_{z}}{a}\left[\sum_{G^{\prime}} W_{\mathrm{l}}\left(G^{\prime}\right)-W_{\mathrm{R}}(0)\right]-\frac{k_{\mathrm{A}}}{a}\left[\sum_{G^{\prime}} W_{\mathrm{l}}\left(G^{\prime}\right)-\sum_{G^{\prime}} W_{\mathrm{u}}\left(G^{\prime}\right)\right] \\
-\omega^{2} m_{\mathrm{R}} W_{\mathrm{R}}(0)=k_{z}\left[\sum_{G^{\prime}} W_{\mathrm{l}}\left(G^{\prime}\right)-2 W_{\mathrm{R}}(0)+\sum_{G^{\prime}} W_{\mathrm{u}}\left(G^{\prime}\right)\right]+2 k_{y}(l-1) W_{\mathrm{R}}(0) \\
\sum_{G^{\prime}}\left\{\left(k+G^{\prime \prime}\right)^{2} m\left(G^{\prime \prime}-G^{\prime}\right)\left(k+G^{\prime}\right)^{2}+\left[1+\mu^{2}\left(k+G^{\prime \prime}\right)^{2}\right] n\left(G^{\prime \prime}-G^{\prime}\right)\left(k+G^{\prime}\right)^{2}\right\} W_{\mathrm{u}}\left(G^{\prime}\right) \\
-\omega^{2} \sum_{G^{\prime}}\left\{\left[1+\mu^{2}\left(k+G^{\prime \prime}\right)^{2}\right] o\left(G^{\prime \prime}-G^{\prime}\right)\right\} W_{\mathrm{u}}\left(G^{\prime}\right) \\
=-\frac{k_{z}}{a}\left[\sum_{G^{\prime}} W_{\mathrm{u}}\left(G^{\prime}\right)-W_{\mathrm{R}}(0)\right]-\frac{k_{\mathrm{A}}}{a}\left[\sum_{G^{\prime}} W_{\mathrm{u}}\left(G^{\prime}\right)-\sum_{G^{\prime}} W_{\mathrm{l}}\left(G^{\prime}\right)\right]
\end{array}\right.
$$

If the number of reciprocal-lattice vectors is picked as $N$, Eq. (16) can be rewritten by a matrix formulation as:

$$
\begin{gathered}
\left(\begin{array}{ccc}
{\left[a[M G]+a[N G]+k_{z}[Q G]+k_{\mathrm{A}}[Q G]\right.} & -k_{\mathrm{z}}[P G]^{\mathrm{T}} & -k_{\mathrm{A}}[Q G] \\
-k_{z}[P G] & 2 k_{z}-2 k_{y}(l-1) & -k_{\mathrm{z}}[P G] \\
-k_{\mathrm{A}}[Q G] & -k_{\mathrm{z}}[P G]^{\mathrm{T}} & a[M G]+a[N G]+k_{\mathrm{z}}[Q G]+k_{\mathrm{A}}[Q G]
\end{array}\right) \\
\quad-\omega^{2}\left[\begin{array}{ccc}
a[O G] & {[0]} & {[0]} \\
{[0]} & m_{\mathrm{R}} & {[0]} \\
{[0]} & {[0]} & a[O G]
\end{array}\right] \\
\quad \times\left[\begin{array}{c}
{\left[W_{\mathrm{l}}(G)\right]} \\
W_{\mathrm{R}}(0) \\
{\left[W_{\mathrm{u}}(G)\right]}
\end{array}\right]=[0]
\end{gathered}
$$

where

$$
\begin{aligned}
& {[M G]_{i j}=\left(k+G_{i}\right)^{2} m\left(G_{i}-G_{j}\right)\left(k+G_{j}\right)^{2}} \\
& {[N G]_{i j}=n\left(G_{i}-G_{j}\right)\left(k+G_{j}\right)^{2}} \\
& {[O G]_{i j}=o\left(G_{i}-G_{j}\right)} \\
& {[P G]=\left[\begin{array}{llll}
1 & 1 & \cdots & 1
\end{array}\right]_{1 \times N}} \\
& {[Q G]=[P G]^{\mathrm{T}}[P G]}
\end{aligned}
$$




$$
\left\{\begin{array}{l}
{\left[W_{1}(G)\right]=\left[\begin{array}{llll}
W_{1}\left(G_{1}\right) & W_{1}\left(G_{2}\right) & \cdots & W_{1}\left(G_{N}\right)
\end{array}\right]^{\mathrm{T}}} \\
{\left[W_{\mathrm{u}}(G)\right]=\left[\begin{array}{llll}
W_{\mathrm{u}}\left(G_{1}\right) & W_{\mathrm{u}}\left(G_{2}\right) & \cdots & W_{\mathrm{u}}\left(G_{N}\right)
\end{array}\right]^{\mathrm{T}}}
\end{array}\right.
$$

Eq. (17) is a typical generalized eigenvalue problem for $\omega^{2}$. By solving the equation for each Bloch wave vector limited in 1BZ, the band structure of the proposed LR piezoelectric/elastic PC Euler double-layer nonlocal nanobeam can be obtained finally.

\section{NUMERICAL RESULTS AND ANALYSES}

\section{A. Complete, symmetric and antisymmetric band structures}

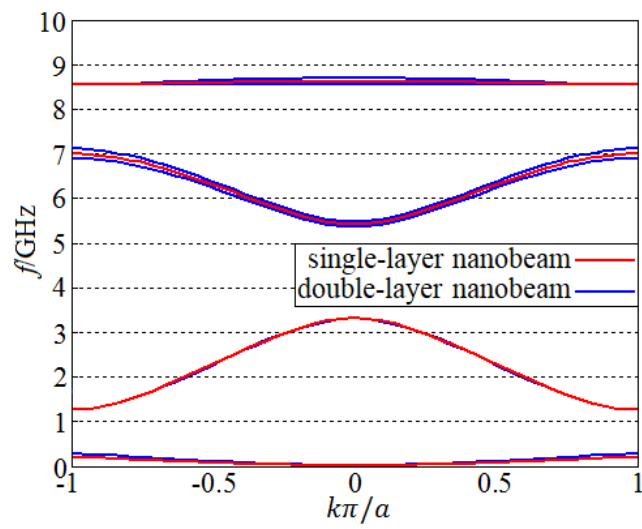

FIG. 2. Band structures of LR piezoelectric/elastic PC Euler single-layer and double-layer nonlocal nanobeams with no horizontal and additional springs attached.

If the stiffness of horizontal spring $k_{y}=0 \mathrm{~N} / \mathrm{m}$ and additional spring $k_{\mathrm{A}}=0 \mathrm{~N} / \mathrm{m}$, the original model is reduced to LR piezoelectric/elastic PC double-layer nonlocal nanobeam with no horizontal and additional springs attached, which the corresponding band structure is shown in Fig. 2. If the upper plate is removed, the LR piezoelectric/elastic PC single-layer nonlocal nanobeam can be obtained, which the corresponding band structure is also shown in Fig. 2 for comparison. During the calculations, $P_{0}=1 \times 10^{-8} \mathrm{~N}, V=1 \mathrm{~V}, \quad m_{\mathrm{R}}=1 \times 10^{-18} \mathrm{~kg}, k_{z}=1 \times 10^{2} \mathrm{~N} / \mathrm{m}$, $\eta=0.1, a_{1}=a_{2}=50 \mathrm{~nm}, b=h=10 \mathrm{~nm}$. As shown in the figure, the addition of upper plate makes the original second, third and fourth bands in single-layer nonlocal nanobeam be divided into two bands. Here, the band gaps of first two orders are researched. For the first band gap, the single-layer nonlocal nanobeam has the lower starting frequency and wider bandgap width. For the second one, the single-layer nonlocal nanobeam has the higher ending frequency and wider bandgap width. Hence, by comparing LR piezoelectric/elastic PC single-layer nonlocal nanobeam with the double-layer one, it seems that single-layer nanobeam always occupies an absolute dominance on opening wider band gaps.

Assuming that the double-layer nanobeam is vibrated in symmetric mode, the constraint conditions $W_{\mathrm{l}}(x)=-W_{\mathrm{u}}(x)$ and $W_{\mathrm{R}}(X)=0$ should be introduced to Eq. (1), then the computational formula of symmetric band structure can be obtained by simplifying Eq. (17) as:

$$
\left(a[M G]+a[N G]+k_{z}[Q G]+2 k_{\mathrm{A}}[Q G]-\omega^{2} a[O G]\right) \times\left[W_{1}(G)\right]=[0]
$$

Assuming that the double-layer nanobeam is vibrated in antisymmetric mode, the constraint condition $W_{\mathrm{l}}(x)=W_{\mathrm{u}}(x)$ should be introduced to Eq. (1), then the computational formula of antisymmetric band structure can be obtained by simplifying Eq. (17) as: 


$$
\begin{gathered}
\left(\left[\begin{array}{cc}
a[M G]+a[N G]+k_{z}[Q G]+k_{\mathrm{A}}[Q G] & -k_{z}[P G]^{\mathrm{T}} \\
-2 k_{z}[P G] & 2 k_{z}-2 k_{y}(l-1)
\end{array}\right]-\omega^{2}\left[\begin{array}{cc}
a[O G] & {[0]} \\
{[0]} & m_{\mathrm{R}}
\end{array}\right)\right. \\
\times\left[\begin{array}{c}
{\left[W_{\mathrm{l}}(G)\right]} \\
W_{\mathrm{R}}(0)
\end{array}\right]=[0]
\end{gathered}
$$

Fig. 3(a) and (b) give the band structures of LR piezoelectric/elastic PC Euler double-layer nonlocal nanobeam with no horizontal and additional springs attached vibrated in symmetric and antisymmetric modes, respectively. As a comparison, the original complete band structure is also displayed. During the calculations, all the parameters are same to those in Fig. 2. As shown, all the bands can be divided into two parts: symmetric and antisymmetric bands. For the bands vibrated in symmetric mode, they cannot be affected by mass because the mass keeps still in such a vibration mode. If the additional springs $k_{\mathrm{A}}$ are added in Fig. 1, the symmetric bands can be adjusted but with the antisymmetric ones unaffected. Besides, if the horizontal springs $k_{y}$ attached onto foundation are added, the antisymmetric bands can be adjusted but with the symmetric ones unaffected.

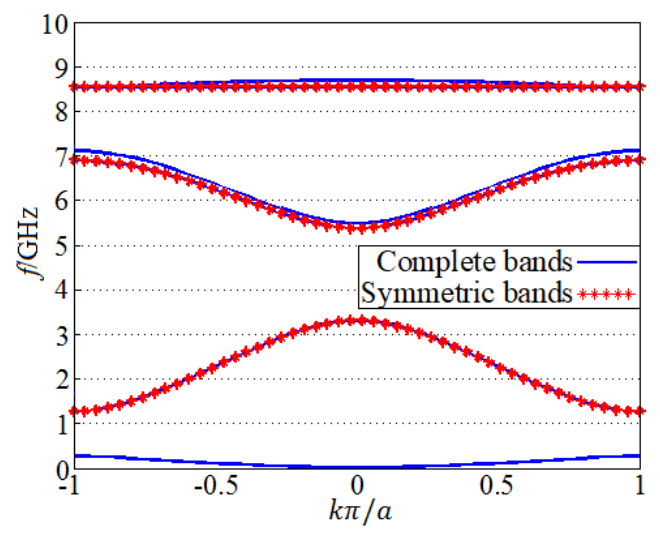

(a)

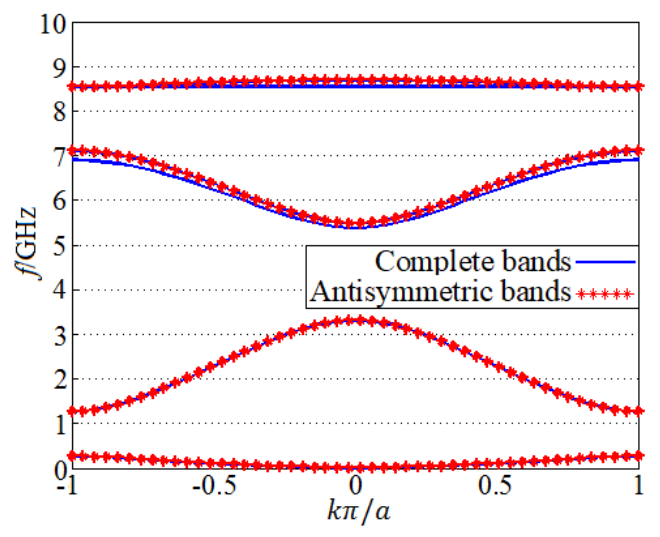

(b)

FIG. 3. (a) Symmetric and (b) antisymmetric band structures of LR piezoelectric/elastic PC Euler double-layer nonlocal nanobeam with no horizontal and additional springs attached.

\section{B. Influences of additional and horizontal springs on band structures}

The band structure of LR piezoelectric/elastic PC Euler double-layer nonlocal nanobeam with additional springs $k_{\mathrm{A}}$ attached is shown in Fig. 4(a). As a comparison, the band structure of the same nanobeam but with no additional springs attached is also displayed, which is divided to symmetric and antisymmetric bands. Here, all the parameters except for $k_{\mathrm{A}}=10 \mathrm{~N} / \mathrm{m}$ are same to those in Fig. 2. As shown, adding additional springs has no influence on antisymmetric bands. However, the symmetric bands are moved upwards to higher frequency region, which can be understood that adding additional springs between upper and lower nanobeams can restrain the symmetric vibration mode and strengthen the equivalent stiffness. As a result, adding additional springs widens the second band gap by increasing the ending frequency. Fig. 4(b) gives the influences of $k_{\mathrm{A}}$ on starting frequencies $f_{\mathrm{s}}$, widths $f_{\mathrm{w}}$ and total width of first two band gaps. Here, all the parameters except for $k_{\mathrm{A}}$ are same to those in Fig. 2, the range of $k_{\mathrm{A}}$ is from $0 \mathrm{~N} / \mathrm{m}$ to $20 \mathrm{~N} / \mathrm{m}$. The starting frequency and width of first band gap keep unchanged with the increase of $k_{\mathrm{A}}$ because the starting and ending frequencies are located at the antisymmetric bands as shown in Fig. 4(a). The starting frequency of second band gap also keeps unchanged with the increase of $k_{\mathrm{A}}$ because it is located at the antisymmetric band as shown in Fig. 4(a). Besides, the width of second band gap keeps increasing firstly and then unchanged with the increase of $k_{\mathrm{A}}$, 
which can be regarded that the ending frequency of such a band gap is located at the symmetric band if $k_{\mathrm{A}} \leq 10 \mathrm{~N} / \mathrm{m}$, but the ending frequency is located at the antisymmetric band if $k_{\mathrm{A}}>$ $10 \mathrm{~N} / \mathrm{m}$ as shown in Fig. 4(a). Hence, the total bandgap width also keeps increasing firstly and then unchanged.

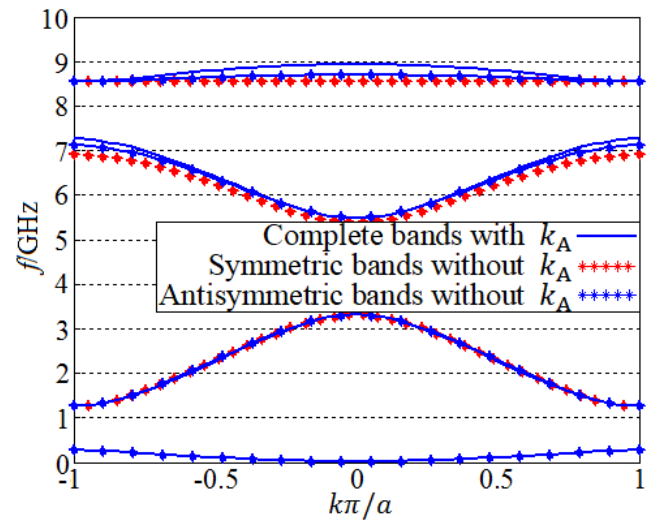

(a)

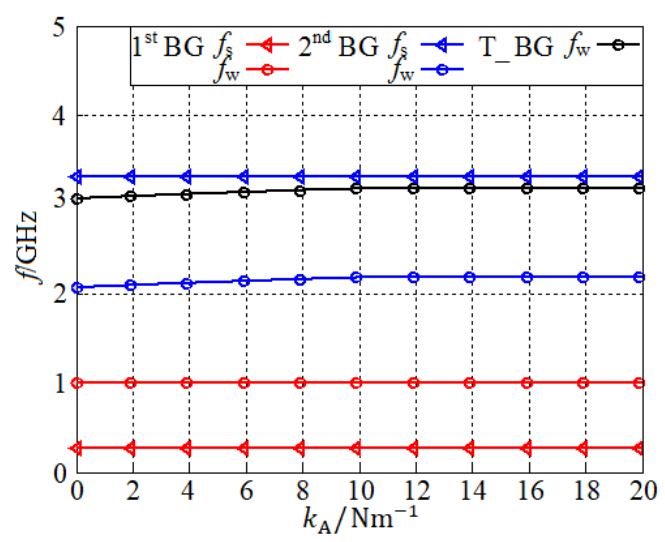

(b)

FIG. 4. (a) Band structures of LR piezoelectric/elastic PC Euler double-layer nonlocal nanobeams with and without additional springs $k_{\mathrm{A}}$ attached, and (b) influences of $k_{\mathrm{A}}$ on starting frequencies $f_{\mathrm{s}}$, widths $f_{\mathrm{w}}$ and total width of first two band gaps.

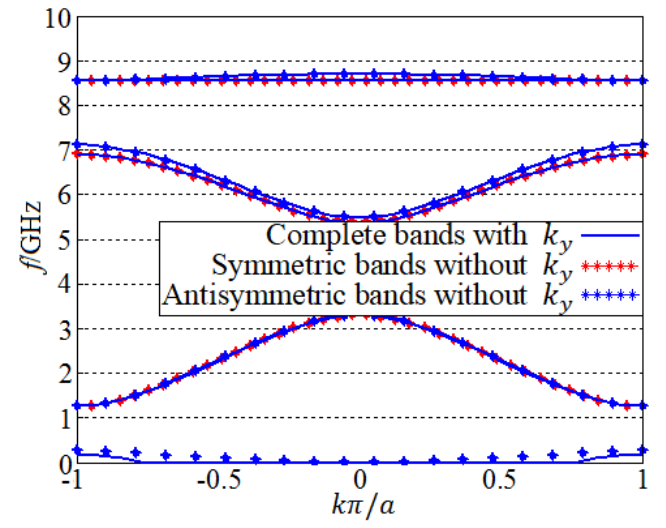

(a)

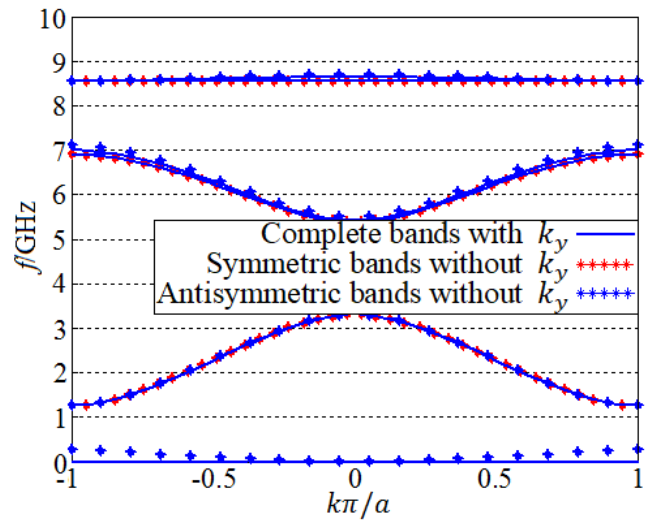

(b)

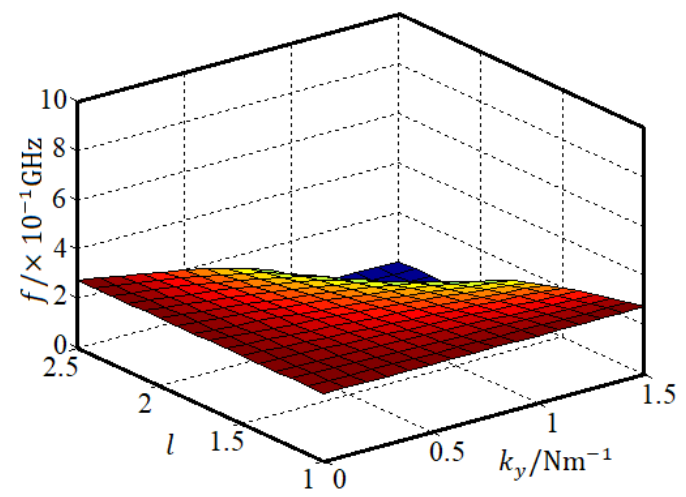

(c)

FIG. 5. Band structures of LR piezoelectric/elastic PC Euler double-layer nonlocal nanobeams with attached horizontal springs (a) $k_{y}=1 \mathrm{~N} / \mathrm{m}$ and (b) $k_{y}=1 \times 10^{3} \mathrm{~N} / \mathrm{m}$, as well as (c) influence of $k_{y}$ and non-dimensional parameter $l$ on starting frequency $f_{\mathrm{s}}$ of first band gap.

Figs. 5(a) and (b) display the band structures of LR piezoelectric/elastic PC Euler 
double-layer nonlocal nanobeams with attached horizontal springs $k_{y}=1 \mathrm{~N} / \mathrm{m}$ and $k_{y}=1 \times$ $10^{3} \mathrm{~N} / \mathrm{m}$, respectively. Here, all the parameters except for $k_{y}$ and non-dimensional parameter $l=2$ are same to those in Fig. 2. As shown, adding horizontal springs has no effect on the symmetric bands because the masses are static in such a vibrational mode. In addition, adding horizontal springs makes the antisymmetric bands move down, which can be understood that the impact of adding pre-compressed horizontal springs is decreasing the original stiffness of vertical springs $k_{z}$. By comparing Figs. 5(a) and (b), only the first band can be affected but with the influence of other antisymmetric bands not obviously if the value of $k_{y}$ is small. Only if the value of $k_{y}$ is big enough, the effect of $k_{y}$ on other antisymmetric bands cannot be ignored. The phenomenon can be attributed to that the value of equivalent stiffness between vertical spring and nanobeam increases with the increase of band order, then larger value of $k_{y}$ is needed to decrease the value of equivalent stiffness. Fig. 5(c) gives the influence of $k_{y}$ and $l$ on starting frequency $f_{\mathrm{s}}$ of first band gap. Here, all the parameters except for $k_{y}$ and $l$ are same to those in Fig. 2, the range of $k_{y}$ is from $0 \mathrm{~N} / \mathrm{m}$ to $1.5 \mathrm{~N} / \mathrm{m}$, and the range of $l$ is from 1 to 2.5 . As shown in the figure, lager value of $k_{y}$ and $l$ can obtain lower frequency of first band gap, which can be regarded that with the increase of $k_{y}$ and $l$, the degree of pre-compression increases, the equivalent stiffness between springs and double-layer nanobeam decreases.

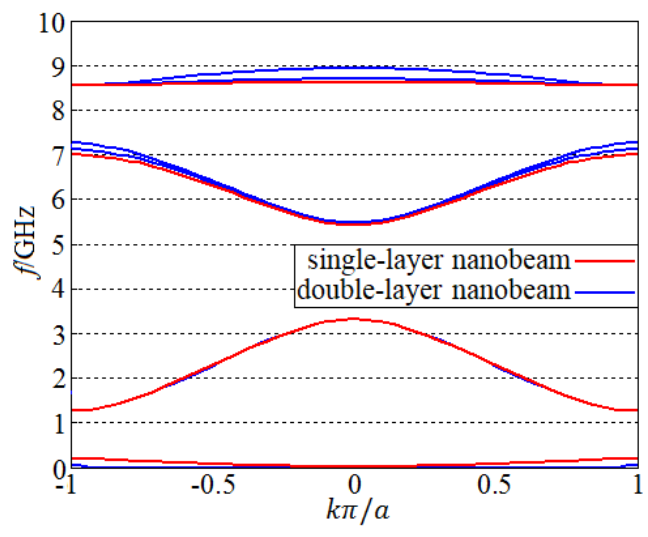

FIG. 6. Band structures of LR piezoelectric/elastic PC Euler single-layer and double-layer nonlocal nanobeams with both horizontal springs $k_{y}$ and additional springs $k_{\mathrm{A}}$ attached.

Fig. 6 displays the band structures of LR piezoelectric/elastic PC Euler single-layer and double-layer nonlocal nanobeams with both horizontal springs $k_{y}$ and additional springs $k_{\mathrm{A}}$ attached. Here, all the parameters except for $l=2, k_{y}=1.5 \mathrm{~N} / \mathrm{m}$ and $k_{\mathrm{A}}=10 \mathrm{~N} / \mathrm{m}$ are same to those in Fig. 2. As shown, for the first band gap, the double-layer nonlocal nanobeam has the lower starting frequency and wider bandgap width than single-layer one. For the second one, the double-layer nonlocal nanobeam has the higher ending frequency and wider bandgap width than single-layer one. By comparing Figs. 6 with 2, adding horizontal and additional springs can widen the band gaps and make the band gaps be controlled, which can be regarded as the advantage that single-layer one doesn't have. Moreover, with the change of resonator parameters, the first symmetric band and second antisymmetric band of double-layer nanobeam are always coincident, which are also coincident with the second band of single-layer nanobeam. The phenomenon can be understood that the position attached resonators in the nanobeams is non-vibrating in such vibration modes corresponding to the bands, which can be further reduced to a single-layer nanobeam with resonators removed.

\section{Influences of parameters on band structures}


The influences of electrical voltage $V$ and axial force $P_{0}$ on starting frequencies $f_{\mathrm{s}}$, widths $f_{\mathrm{w}}$ and total width of first two band gaps are shown in Figs. 7(a) and (b), respectively. Here, all the parameters except for $V$ and $P_{0}$ are same to those in Fig. 6. The range of $V$ is from $-1 \mathrm{~V}$ to $1 \mathrm{~V}$, and the range of $P_{0}$ is from $1 \times 10^{-8} \mathrm{~N}$ to $5 \times 10^{-8} \mathrm{~N}$. With the increase of $V$, both the starting frequencies of first two band gaps keep decreasing and both the widths of first two band gaps keep increasing. Finally, the total width of first two band gaps keeps increasing by increasing $V$. With the increases of $P_{0}$, both the starting frequencies of first two band gaps keep increasing, the width of first band gap keeps increasing and the second one keeps decreasing. Finally, the total width of first two band gaps keeps decreasing by increasing $P_{0}$.

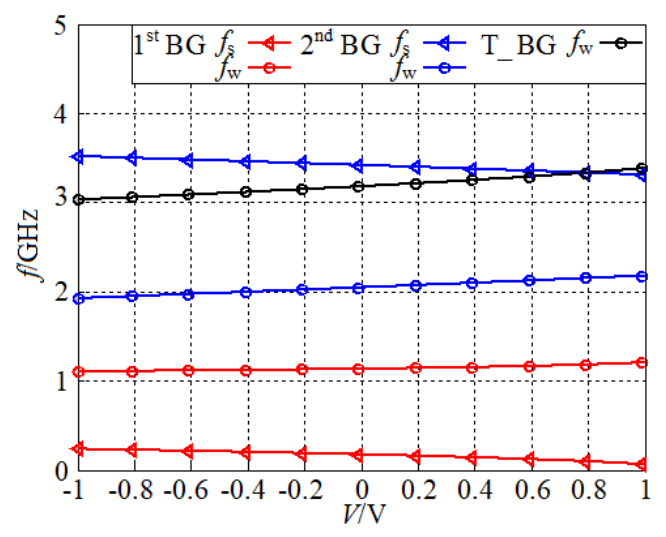

(a)

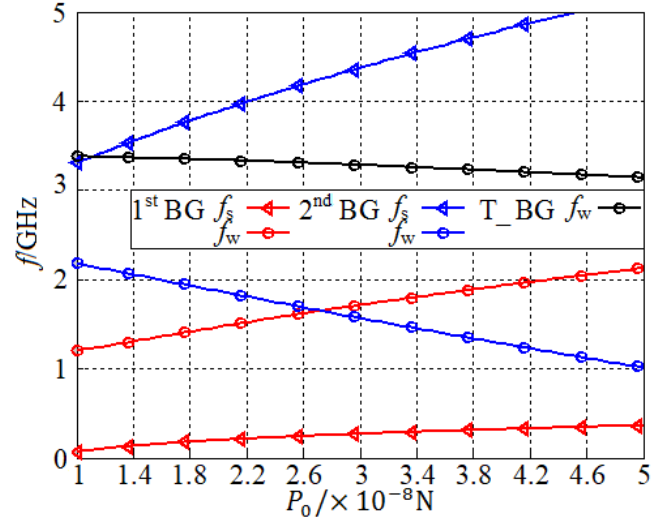

(b)

FIG. 7. Influences of (a) electrical voltage $V$ and (b) axial force $P_{0}$ on starting frequencies $f_{\mathrm{s}}$, widths $f_{\mathrm{w}}$ and total width of first two band gaps, respectively.

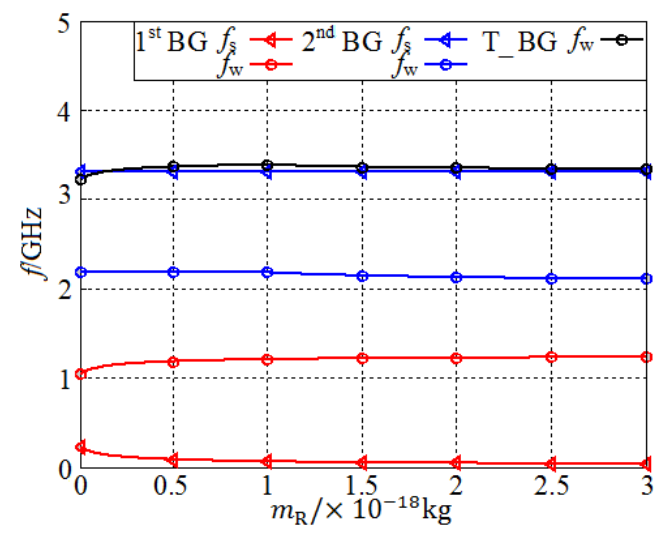

(a)

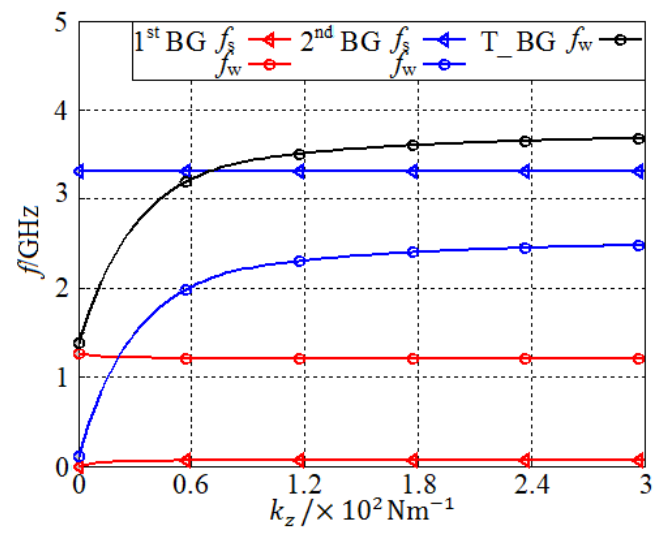

(b)

FIG. 8. Influences of (a) mass $m_{\mathrm{R}}$ and (b) vertical spring $k_{z}$ on starting frequencies $f_{\mathrm{s}}$, widths $f_{\mathrm{w}}$ and total width of first two band gaps, respectively.

The influences of mass $m_{\mathrm{R}}$ and vertical spring $k_{z}$ on starting frequencies $f_{\mathrm{s}}$, widths $f_{\mathrm{w}}$ and total width of first two band gaps are shown in Figs. 8(a) and (b), respectively. Here, all the parameters except for $m_{\mathrm{R}}$ and $k_{z}$ are same to those in Fig. 6. The range of $m_{\mathrm{R}}$ is from $0 \mathrm{~kg}$ to $3 \times 10^{-18} \mathrm{~kg}$, and the range of $k_{z}$ is from $0 \mathrm{~N} / \mathrm{m}$ to $3 \times 10^{2} \mathrm{~N} / \mathrm{m}$. As shown in Fig. 8(a), with the increase of $m_{\mathrm{R}}$, the starting frequency of first band gap keeps decreasing, which can be understood that the equivalent mass of resonators increases by increasing $m_{R}$. The ending frequency is located at the symmetric band as shown in Fig. 3(a) that unaffected by mass, which leads the width of first band gap to keep increasing. With the increase of $m_{\mathrm{R}}$, the starting frequency of second band gap keeps unchanged because it is also located at the symmetric band as 
shown in Fig. 3(a). The width of second band gap keeps static firstly and then decreasing by increasing $m_{\mathrm{R}}$, which can be regarded that the ending frequency of second band gap is also located at the symmetric band if $m_{\mathrm{R}} \leq 1 \times 10^{-18} \mathrm{~kg}$, and it is also located at the antisymmetric band if $m_{\mathrm{R}}>1 \times 10^{-18} \mathrm{~kg}$. Finally, the total width of first two band gaps keeps increasing firstly and then decreasing with the increase of $m_{\mathrm{R}}$. Moreover, as shown in Fig. 8(b), with the increase of $k_{z}$, the ending frequency of first band gap and starting frequency of second band gap keep unchanged because they are located at the bands with resonators ineffective, which is revealed in the above section. Besides, with the increase of $k_{z}$, the starting frequency of first band gap and sending frequency of second band gap keep increasing because the equivalent stiffness of resonators increases by increasing $k_{z}$. Finally, with the increase of $k_{z}$, the width of first band gap keeps decreasing, the second and total ones keep increasing.

The influences of non-dimensional parameters $a_{1} / a_{2}$ and $h / b$ on starting frequencies $f_{\mathrm{s}}$, widths $f_{\mathrm{w}}$ and total width of first two band gaps are shown in Figs. 9(a) and (b), respectively. Here, all the parameters except for $a_{1}$ and $h$ are same to those in Fig. 6. The range of $a_{1} / a_{2}$ is from 0 to 4 , and the range of $h / b$ is from 0.5 to 2. As shown in Fig. 9(a), with the increase of $a_{1} / a_{2}$, the starting frequency of first band gap keeps decreasing to zero, the width of first band gap keeps decreasing, the starting frequency of second band gap keeps decreasing, and the width of second band gap keeps decreasing after a down and up. Finally, the total width of first two band gaps keeps decreasing after a down and up by increasing $a_{1} / a_{2}$. As shown in Fig. 9(b), with the increase of $h / b$, the starting frequency of first band gap is zero firstly and then keeps increasing, the width of first band gap keeps increasing firstly and then decreasing, the starting frequency of second band gap keeps increasing, and the width of second band gap keeps increasing firstly and then decreasing. Finally, the total width of first two band gaps keeps increasing firstly and then decreasing by increasing $h / b$. Hence, the values of $a_{1} / a_{2}$ and $h / b$ corresponding to the peaks shown in Figs. 9(a) and (b) can be considered to make the band gaps as wide as possible during the process of design.

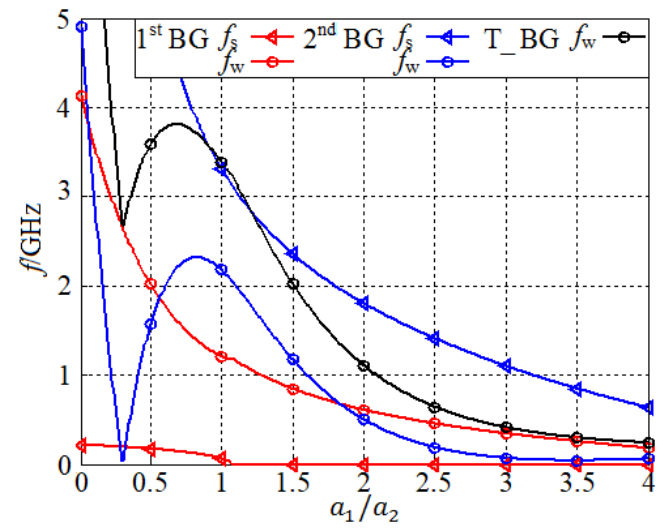

(a)

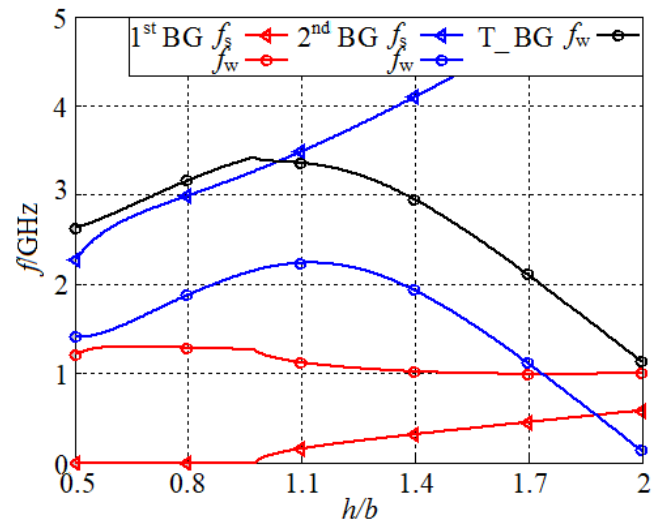

(b)

FIG. 9. Influences of non-dimensional parameters (a) $a_{1} / a_{2}$ and (b) $h / b$ on starting frequencies $f_{\mathrm{s}}$, widths $f_{\mathrm{w}}$ and total width of first two band gaps, respectively.

The influences of non-dimensional parameter $\eta$ on starting frequencies $f_{\mathrm{s}}$, widths $f_{\mathrm{w}}$ and total width of first two band gaps is shown in Figs. 10. Here, all the parameters except for $\eta$ are same to those in Fig. 6. The range of $\eta$ is from 0 to 0.5 . As shown, with the increase of $\eta$, the starting frequency of first band gap is zero firstly, then keeps increasing and finally decreasing to zero, the width of first band gap keeps decreasing, the starting frequency of second band gap 
keeps decreasing, and the width of second band gap keeps increasing firstly and then decreasing. Finally, the total width of first two band gaps keeps increasing firstly and then decreasing by increasing $\eta$.

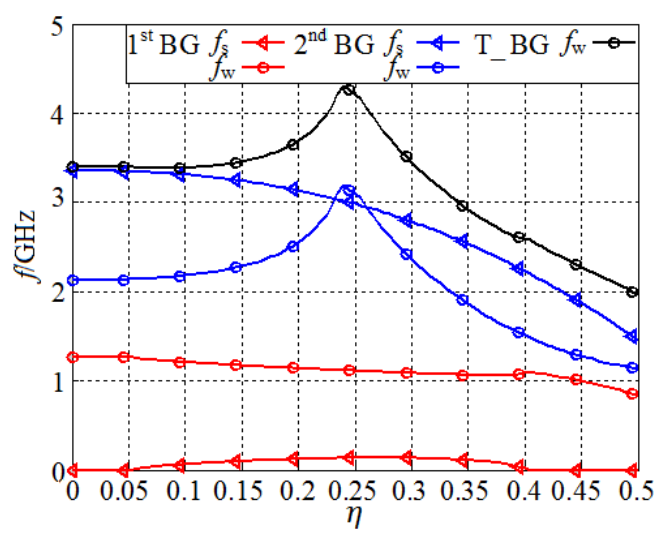

FIG. 10. Influences of non-dimensional parameter $\eta$ on starting frequencies $f_{\mathrm{s}}$, widths $f_{\mathrm{w}}$ and total width of first two band gaps.

\section{CONCLUSIONS}

In this paper, the band structure of a proposed LR piezoelectric/elastic PC Euler double-layer nonlocal nanobeam with horizontal and additional springs periodically attached is calculated based on PWE method. The main properties of band gaps are reveled as follows:

1. If horizontal and additional springs are not attached, the corresponding single-layer nanobeam can open wider band gaps than double-layer one. However, if horizontal and additional springs are attached, double-layer nanobeam is better with the whole quality of nanobeam not increased.

2. All the bands of double-layer nanobeam can be divided into symmetric and antisymmetric ones. Adding additional springs can effectively control the symmetric bands, and adding horizontal springs can effectively adjust the antisymmetric bands. By increasing the stiffness of additional spring, the width of second band gap increases. By increasing the stiffness of horizontal spring or non-dimensional pre-compressed parameter, the width of first band gap increases.

3. The starting frequencies and widths of band gaps can be effectively controlled by electrical voltage, axial force and the parameters of resonator, which can be further applied to realize the active control of vibration. Moreover, the influence rules of geometric parameters and non-dimensional nonlocal parameter on band gaps are also revealed.

\section{Availability of data and materials}

Not applicable.

\section{Competing interests}

The authors declare that they have no competing interests.

\section{Funding}

Supported by National Natural Science Foundation of China (Grant Nos. 11847009, 51979130).

\section{Authors' contributions}

DQ proposed the model and numerical method, JZ and JW analyzed the data, all the authors wrote and improved the paper. All authors read and approved the final manuscript.

\section{Acknowledgements}


Not applicable.

\section{REFERENCES}

[1] Yildirim T, Zhang L, Neupane G P, et al. Towards future physics and applications via two-dimensional material NEMS resonators[J]. Nanoscale, 2020, 12(44): 22366-22385.

[2] Pereira F N, Santos J M C D. Phononic crystal investigation using a fluid-structure circular cylindrical shell spectral element[J]. Mechanical Systems and Signal Processing, 2021, 148: 107100.

[3] Wang $\mathrm{H}$, Lee $\mathrm{H} \mathrm{P}, \mathrm{Xu} \mathrm{W}$. Bandgap properties of two-layered locally resonant phononic crystals[J]. International Journal of Applied Mechanics, 2020, 12(7): 2050075.

[4] Yan H B, Yang X H, Lu T J, et al. Convective heat transfer in a lightweight multifunctional sandwich panel with X-type metallic lattice core[J]. Applied Thermal Engineering, 2017, 127: 1293-1304.

[5] Chen C M, Guo Z F, Liu S T, et al. Effect of cavity structure on acoustic characteristics of phononic crystals based on double-layer plates[J]. Crystals, 2020, 10(11): 995.

[6] Yan D J, Chen A L, Wang Y S, et al. Propagation of guided elastic waves in nanoscale layered periodic piezoelectric composites[J]. European Journal of Mechanics A/Solids, 2017, 66: 158-167.

[7] Yan D J, Chen A L, Wang Y S, et al. In-plane elastic wave propagation in nanoscale periodic layered piezoelectric structures[J]. International Journal of Mechanical Sciences, 2018, 142-143: 276-288.

[8] Chen A L, Yan D J, Wang Y S, et al. Anti-plane transverse waves propagation in nanoscale periodic layered piezoelectric structures[J]. Ultrasonics, 2016, 65: 154-164.

[9] Miranda Jr E J P, Dos Santos J M C. Complete band gaps in nano-piezoelectric phononic crystals[J]. Materials Research, 2017, 20: 15-38.

[10] Qian D H. Bandgap properties of a piezoelectric phononic crystal nanobeam based on nonlocal theory[J]. Journal of Materials Science, 2019, 54(5): 4038-4048.

[11] Qian D H. Bandgap properties of a piezoelectric phononic crystal nanobeam with surface effect[J]. Journal of Applied Physics, 2018, 124(5): 055101.

[12] Qian D H, Shi Z Y, Ning C W, et al. Nonlinear bandgap properties in a nonlocal piezoelectric phononic crystal nanobeam[J]. Physics Letters A, 2019, 383(25): 3101-3107.

[13] Qian D H. Electro-mechanical coupling wave propagating in a locally resonant piezoelectric/elastic phononic crystal nanobeam with surface effects[J]. Applied Mathematics and Mechanics (English Edition), 2020, 41(3): 425-438.

[14] Zeng S, Wang B L, Wang K F. Static stability analysis of nanoscale piezoelectric shells with flexoelectric effect based on couple stress theory[J]. Microsystem Technologies, 2018: 1-11.

[15] Zhou Y, Xu Y, Pan D, et al. Improved incorporation of strain gradient elasticity in the flexoelectricity based energy harvesting from nanobeams[J]. Physica E: Low-dimensional Systems and Nanostructures, 2018, 98: 148-158.

[16] Arefi M, Rabczuk T. A nonlocal higher order shear deformation theory for electro-elastic analysis of a piezoelectric doubly curved nano shell[J]. Composites Part B: Engineering, 2019, 168: 496-510.

[17] Ansari R, Oskouie M F, Gholami R, et al. Thermo-electro-mechanical vibration of post 
buckled piezoelectric Timoshenko nanobeams based on the nonlocal elasticity theory[J]. Composites Part B, 2016, 89: 316-327.

[18] Liu P, Zuo S, Wu X, et al. Study on the vibration attenuation property of one finite and hybrid piezoelectric phononic crystal beam[J]. European Journal of Mechanics-A/Solids, 2020, 84: 104017.

[19] Qi X Q, Li T J, Zhang J L. Tuning characteristics of bandgap in a stub phononic crystal plate with slit and slider structure[J]. Materials Research Express, 2019, 6(12): 126206.

[20] Wang L Q, Zheng H, Zhao M L, et al. Petrov-Galerkin method for the band structure computation of anisotropic and piezoelectric phononic crystals[J]. Applied Mathematical Modelling, 2021, 89: 1090-1105.

[21] Deng T, Zhang S Z, Gao Y W. A magnetic-dependent vibration energy harvester based on the tunable point defect in 2D magneto-elastic phononic crystals[J]. Crystal, 2019, 9(5): 261.

[22] Guo X, Wei P, Lan M, et al. Dispersion relations of elastic waves in one-dimensional piezoelectric/piezomagnetic phononic crystal with functionally graded interlayers.[J]. Ultrasonics, 2016, 70: 158-171.

[23] Yan Z, Wei C, Zhang C. Band structures of elastic SH waves in nanoscale multi-layered functionally graded phononic crystals with/without nonlocal interface imperfections by using a local RBF collocation method[J]. Acta Mechanica Solida Sinica, 2017, 30(4): 390-403.

[24] Hosseini S M, Zhang C Z. Nonlocal coupled thermoelastic wave propagation band structures of nano-scale phononic crystal beams based on GN theory with energy dissipation: An analytical solution[J]. Wave Motion, 2020, 92: 102429.

[25] Xiao Y, Wen J, Wen X. Flexural wave band gaps in locally resonant thin plates with periodically attached spring-mass resonators[J]. Journal of Physics D Applied Physics, 2012, 45(19): 195401-195412(12). 
Figures

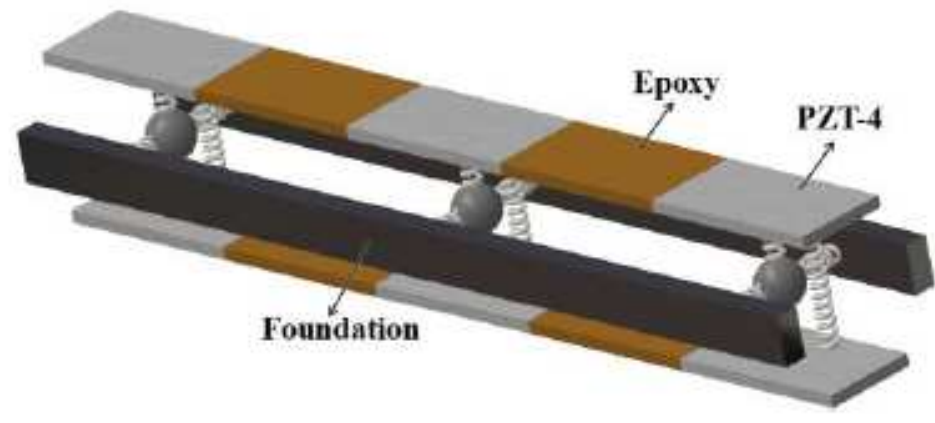

(a)

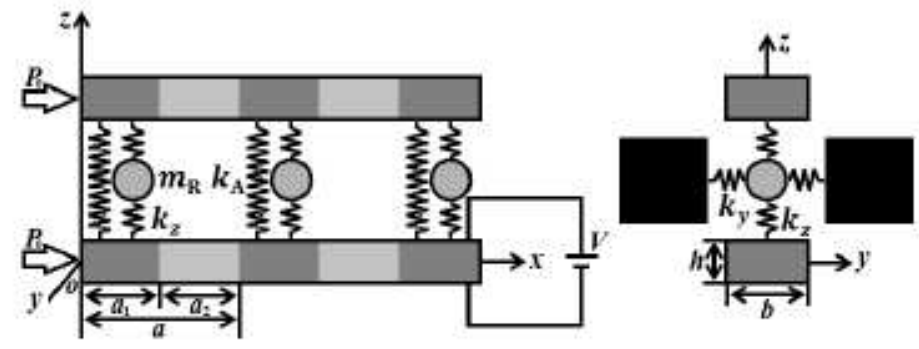

(b)

\section{Figure 1}

See the Manuscript Files section for the complete figure caption.

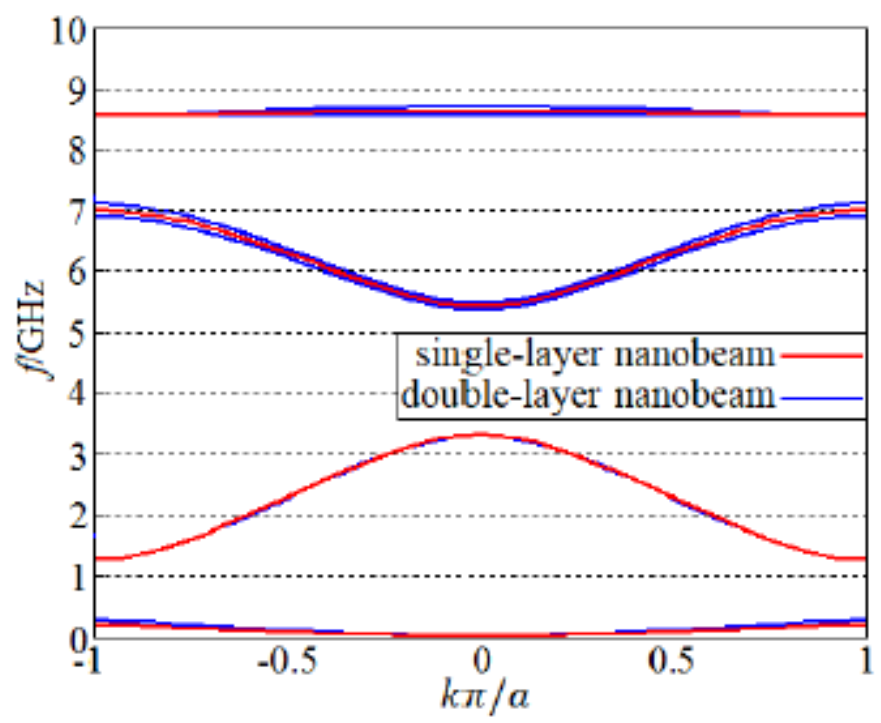

Figure 2

See the Manuscript Files section for the complete figure caption. 


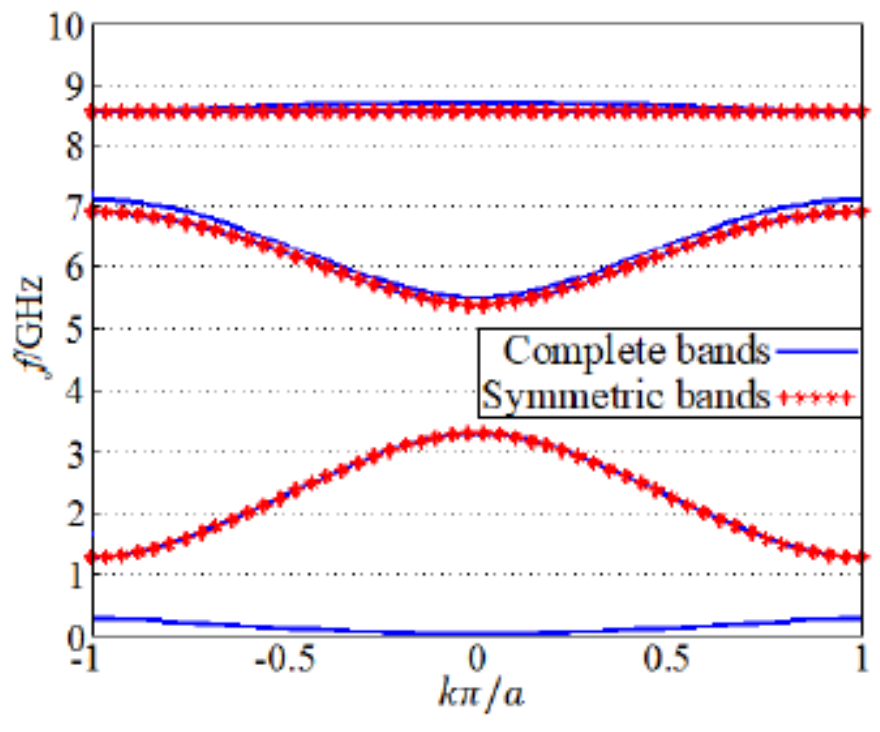

(a)

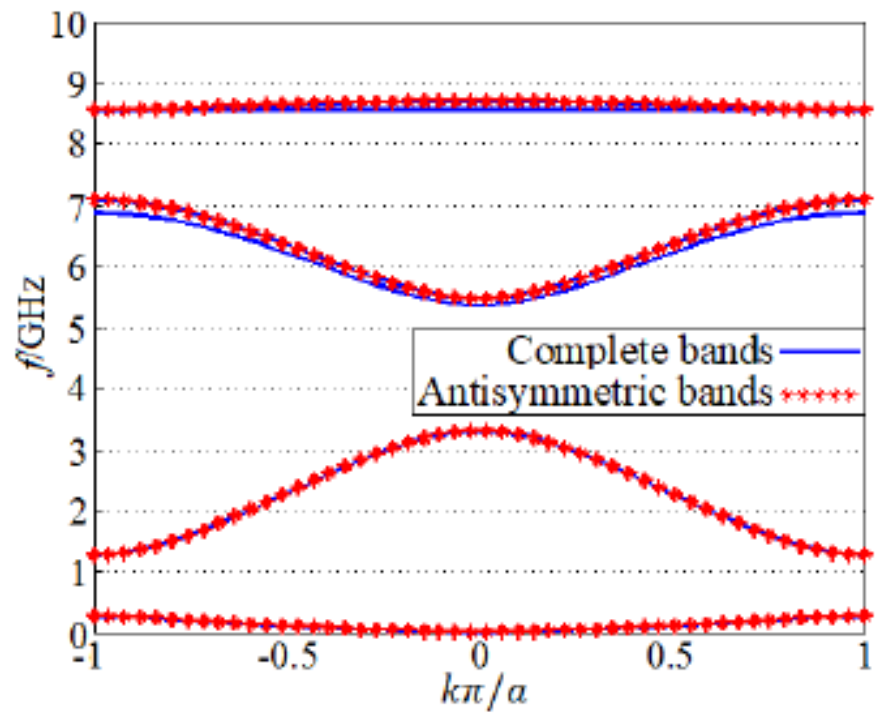

(b)

\section{Figure 3}

See the Manuscript Files section for the complete figure caption.

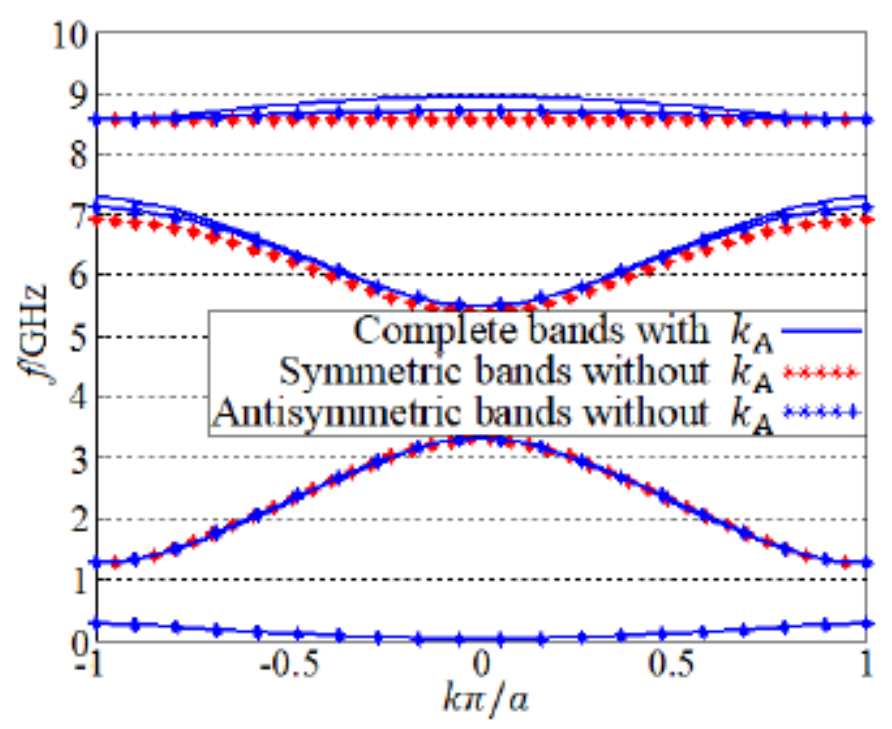

(a)

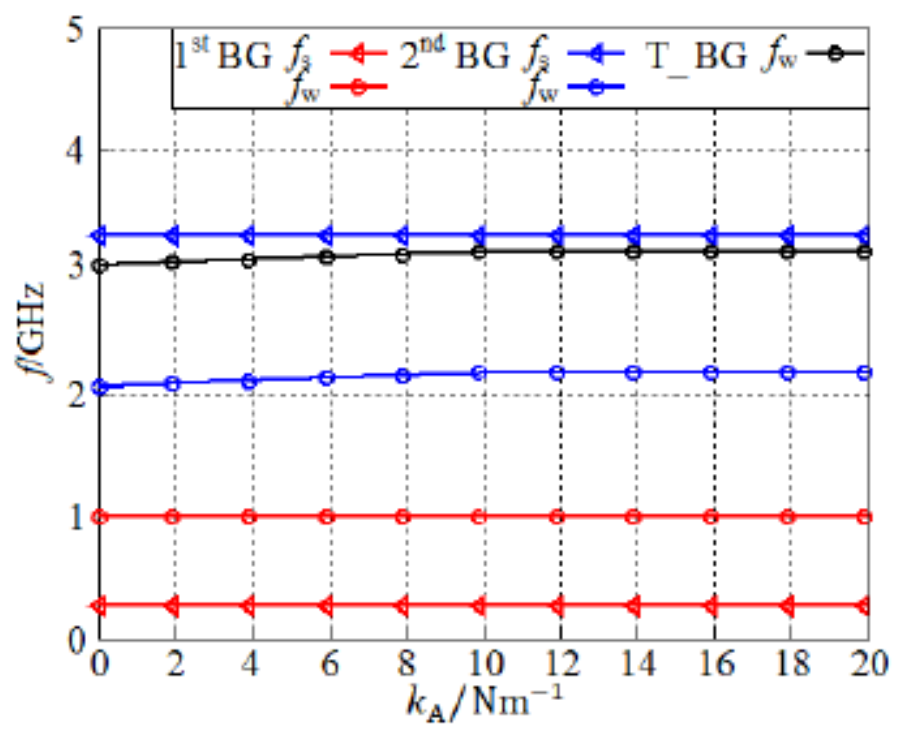

(b)

\section{Figure 4}

See the Manuscript Files section for the complete figure caption. 


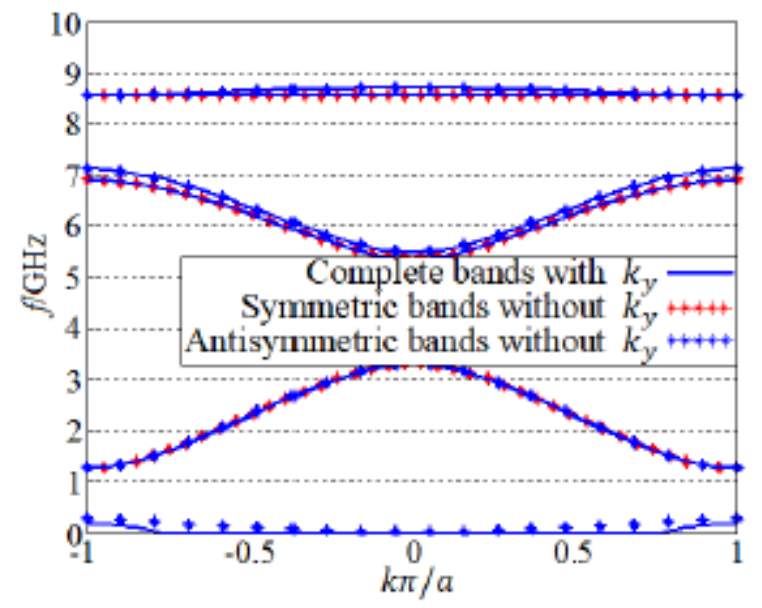

(a)

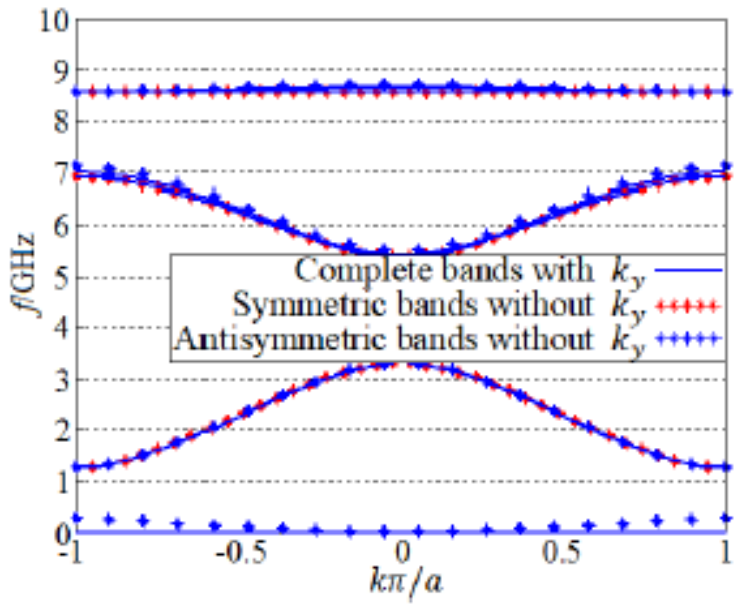

(b)

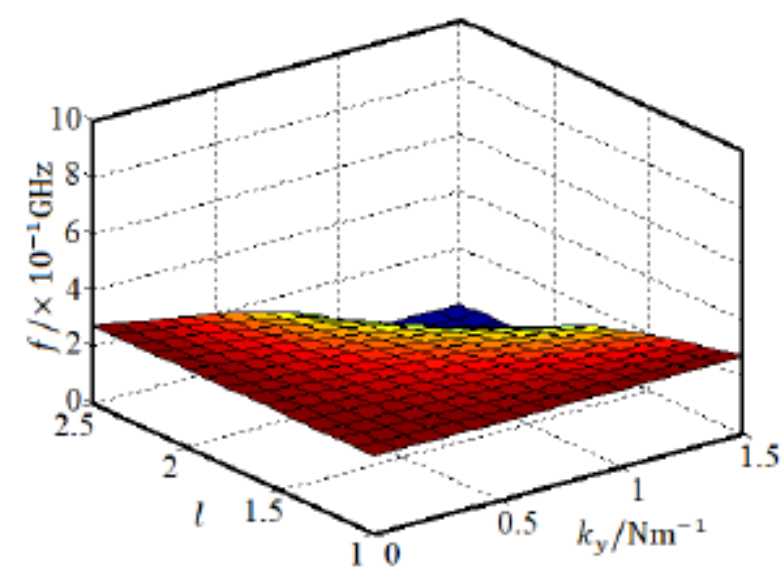

(c)

\section{Figure 5}

See the Manuscript Files section for the complete figure caption.

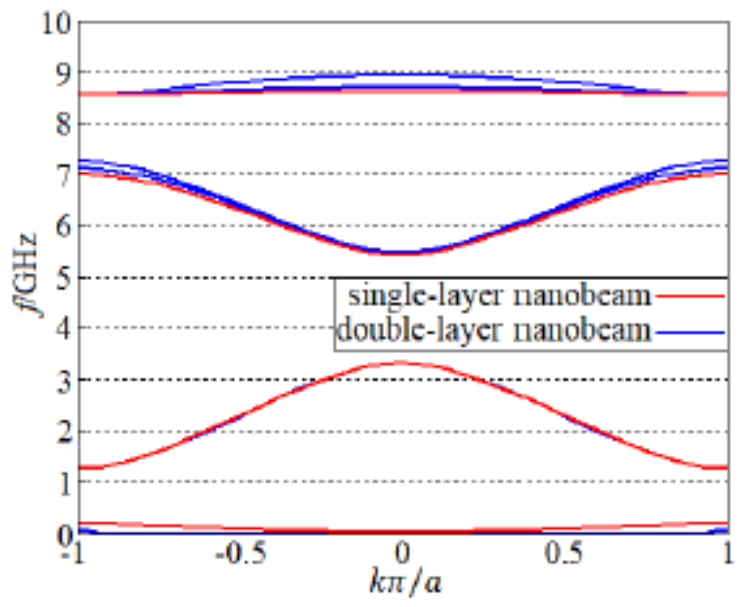

Figure 6

See the Manuscript Files section for the complete figure caption. 


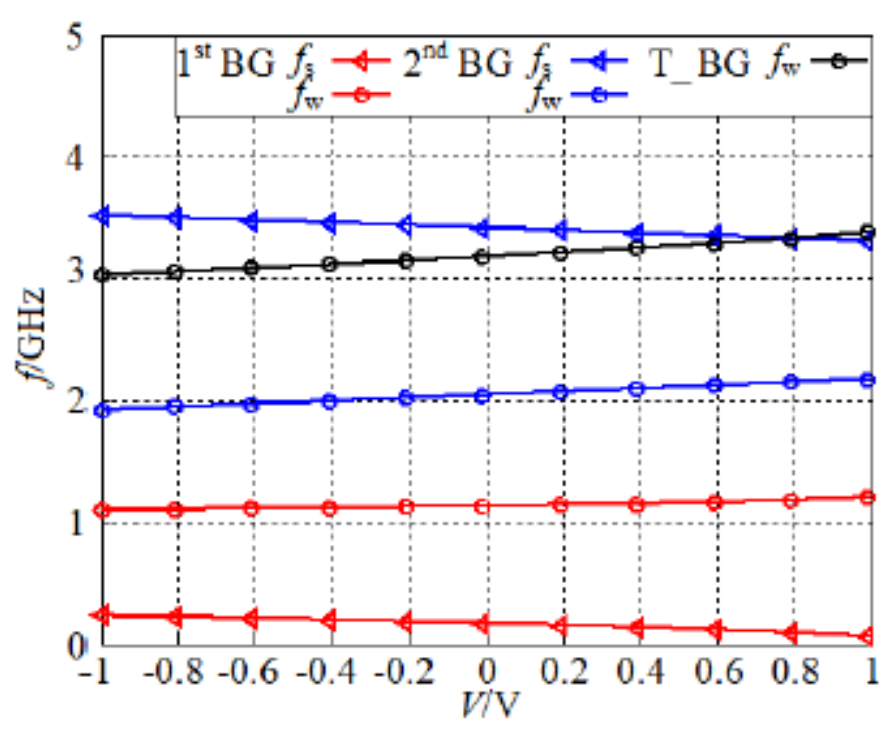

(a)

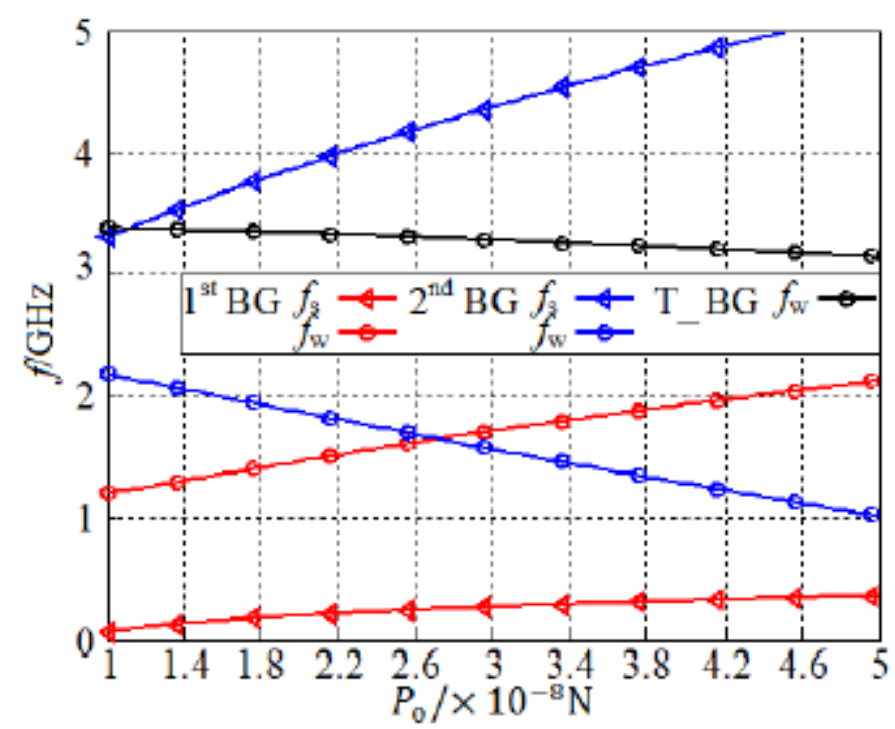

(b)

Figure 7

See the Manuscript Files section for the complete figure caption.

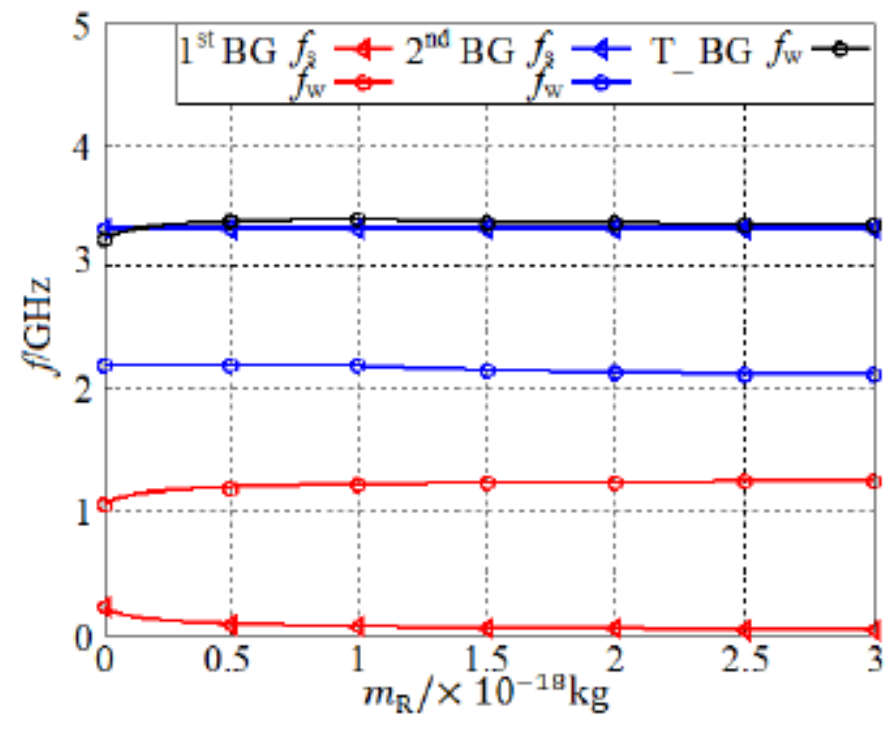

(a)

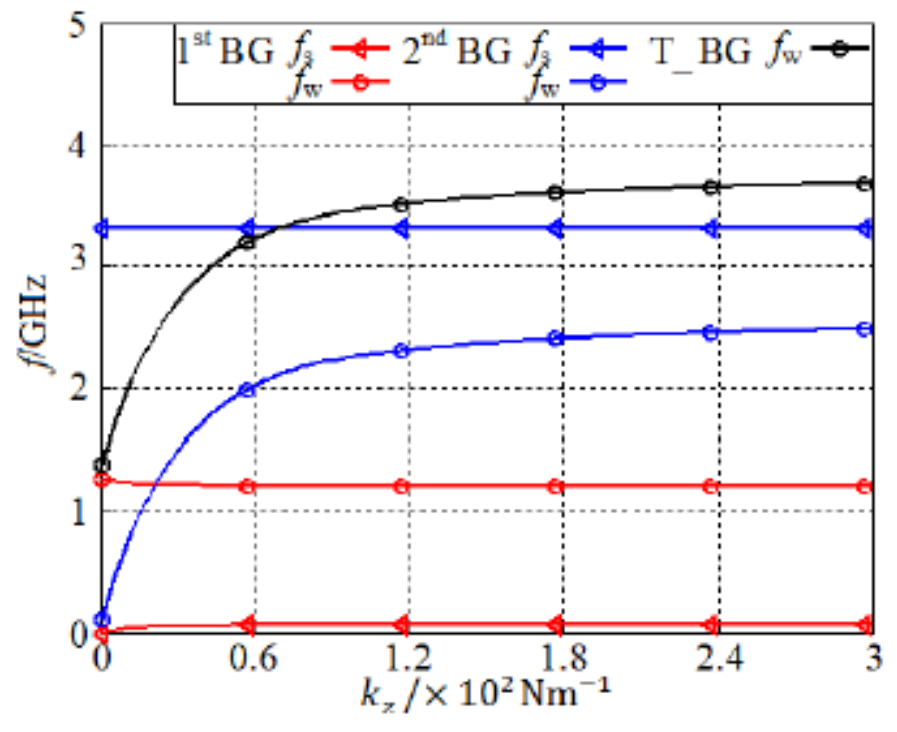

(b)

Figure 8

See the Manuscript Files section for the complete figure caption. 


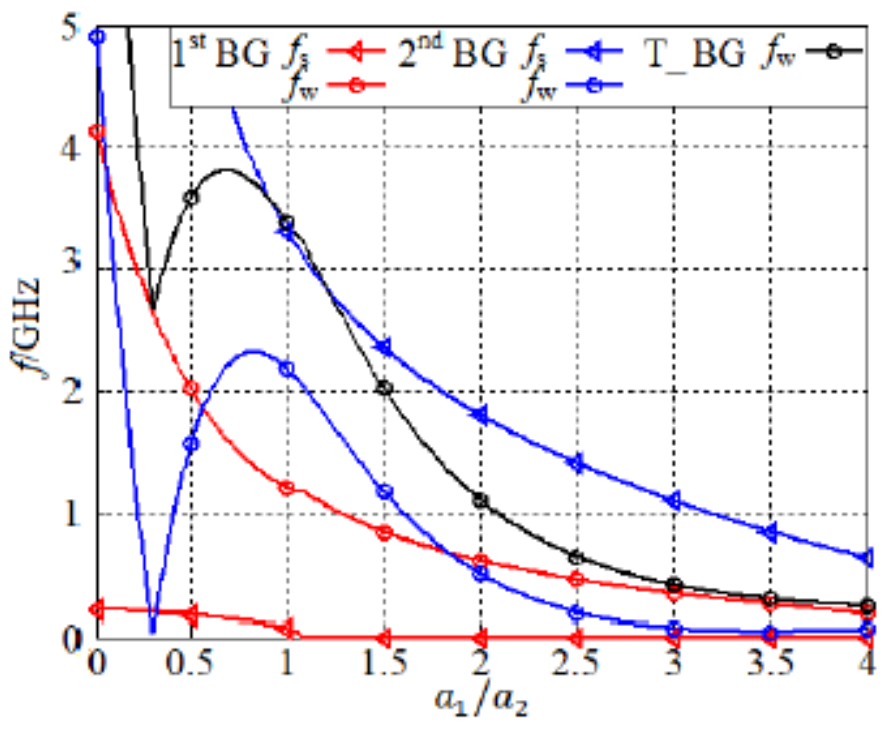

(a)

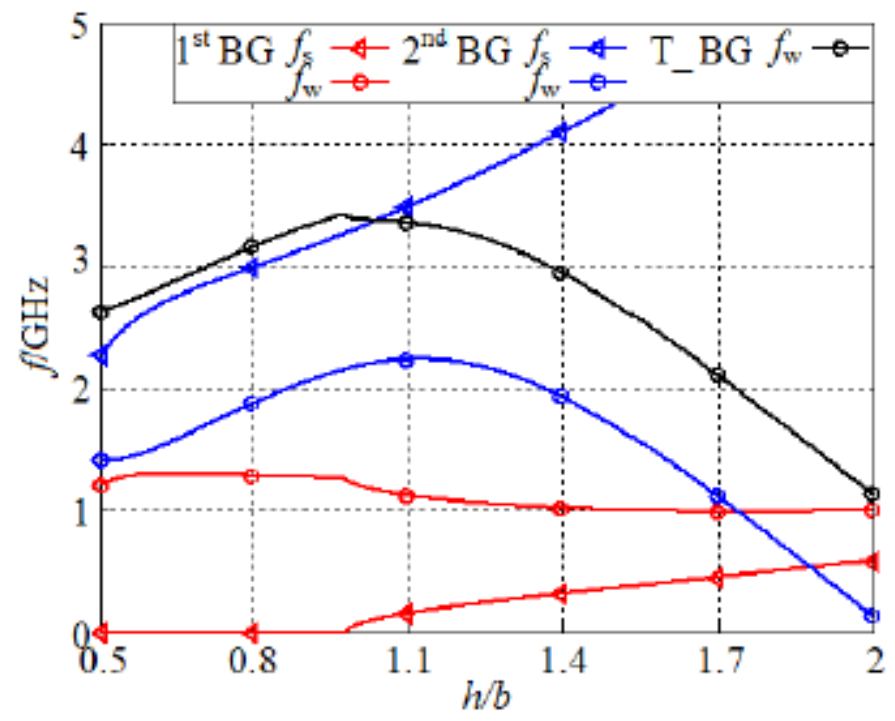

(b)

Figure 9

See the Manuscript Files section for the complete figure caption.

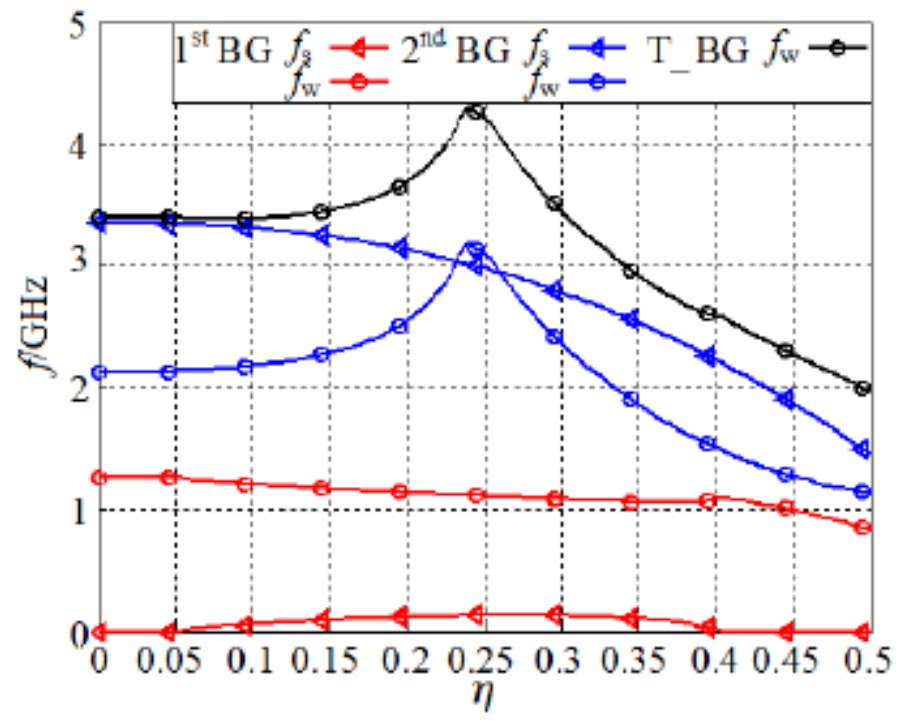

Figure 10

See the Manuscript Files section for the complete figure caption. 